\title{
Emancipação na luta pela equidade em pesquisas com seres humanos
}

Dirceu B. Greco ${ }^{1}$

\begin{abstract}
Resumo
Este artigo apresenta como estudo de caso os aspectos mais controversos de pesquisa conduzida na Guatemala (1946-1948), patrocinada pelo Serviço de Saúde Pública dos Estados Unidos e envolvendo participantes infectados intencionalmente pelo Treponema pallidum. Analisando as alterações de 2008 na Declaração de Helsinque e a ainda pequena adesão a outros instrumentos internacionais, como a Declaração Universal sobre Bioética e Direitos Humanos, da Unesco, a discussão enfatiza a importância da implantação de diretrizes éticas internacionalmente aceitas para impedir que voluntários humanos sejam envolvidos em pesquisas não éticas. Conclui considerando que é indispensável o envolvimento de cientistas e ativistas para alcançar padrões éticos universalmente aceitos e aplicáveis, visando evitar duplo standard. Além disso, que a participação igualitária em pesquisas e distribuição justa de seus benefícios será importante passo para atingir o acesso universal a cuidados de saúde de qualidade para todos.

Palavras-chave: Ética em pesquisa. Saúde pública. Declaração de Helsinque. Proteção. Efetividade.
\end{abstract}

\section{Resumen}

\section{Emancipación en la lucha por la equidad en investigaciones con seres humanos}

Este artículo presenta como estudio de caso los aspectos más controvertidos de la investigación realizadas en Guatemala (1946-1948), patrocinada por el Servicio de Salud Pública de los Estados Unidos e involucrando participantes infectados por el Treponema pallidum por todo el mundo. Analizando los cambios en la Declaración de Helsinki de 2008 y la pequeña adhesión, en aquel entonces, a directrices internacionales como la Declaración Universal sobre Bioética y Derechos Humanos de UNESCO la discusión enfatiza la importancia de aplicar normas éticas aceptadas internacionalmente para impedir que voluntarios humanos participen de investigaciones no éticas y concluye considerando que es indispensable la participación de científicos y activistas para alcanzar las normas éticas universalmente aceptados y aplicables, para evitar el doble estándar. Además, que la participación igualitaria en investigaciones y la distribución justa de sus beneficios será un importante paso para lograr el acceso universal a cuidados de una salud de calidad para todos.

Palabras-clave: Ética en Investigación. Salud pública. Declaración de Helsinki. Protección. Efectividad.

\section{Abstract \\ Emancipation in the struggle for equality in research involving human volunteers}

This manuscript presents as a case study the most controversial aspects of a research conducted in Guatemala (1946-1948), sponsored by the United States Public Health Service in which participants were intentionally infected with Treponema pallidum. The analysis of changes in the 2008 Declaration of Helsinki and the still insufficient adherence to UNESCO's Universal Declaration on Bioethics and Human Rights emphasizes the importance of applying internationally accepted ethical standards for human volunteers to prevent unethical research. It concludes that the involvement of scientists and activists is crucial to achieve internationally accepted ethical standards to be equally applied throughout the world, avoiding the risks of double standards, and also that egalitarian participation in research and fair distribution of its benefits will be an important step towards universal access to good quality healthcare for all people.

Key words: Ethics, research. Public health. Helsinki Declaration. Protection. Effectiveness.

1. Pós-doutor dirceugreco@gmail.com - Departamento de Clínica Médica, Faculdade de Medicina, Universidade Federal de Minas Gerais (UFMG), Belo Horizonte/MG, Brasil.

\section{Correspondência}

Serviço de Doenças Infecciosas e Parasitárias, Faculdade de Medicina e Hospital das Clínicas Av. Alfredo Balena, 190, $1^{\circ}$ andar CEP 30130-100. Belo Horizonte/MG, Brasil.

Declara não haver conflito de interesse. 
A discussão sobre as normas éticas para pesquisas biomédicas em países em desenvolvimento ganhou considerável visibilidade nos últimos anos ${ }^{1-4}$. Este interesse foi inicialmente alimentado pela necessidade de pesquisas relacionadas à pandemia da aids e pela migração de ensaios clínicos dos países industrializados para os países em desenvolvimento. Embora algumas das doenças pesquisadas ocorram globalmente, o que parcialmente explicaria a inclusão dos países em desenvolvimento, é preocupante que este aumento não inclua as variadas doenças que afetam especialmente os países mais pobres.

Mesmo com o progresso da ciência e da tecnologia, os benefícios raramente alcançam estas populações. Mais de um terço da população mundial ainda vive em condições intoleráveis de pobreza, com pouco ou nenhum acesso aos serviços de saúde e aos medicamentos mais básicos. Assim, para que o progresso científico acompanhe o progresso moral, é necessário que os padrões dos serviços de saúde melhorem e os investimentos sejam direcionados, preferencialmente, ao desenvolvimento de estudos éticos e relevantes às prioridades locais, visando à melhoria da saúde pública para todos ${ }^{5}$.

O registro de diversas pesquisas não éticas envolvendo seres humanos, não somente durante a $2^{\text {a }}$ Guerra Mundial ${ }^{6}$, mas em tempos teoricamente pacíficos, como as atrocidades em Tuskegee ${ }^{7,8}$ e as barbaridades recentemente reveladas de pesquisa na Guatemala (1946-1948) 9-11, demonstram a importância desta discussão, que será o foco deste artigo. Ao apresentar e analisar esses casos se pretende ilustrar os principais impasses que, ainda atualmente, afetam o controle ético de estudos clínicos em todo o mundo.

\section{A ética na pesquisa internacional e na saúde global}

É indubitável a necessidade de desenvolver vacinas eficazes e medicamentos potentes e acessíveis para o tratamento ou controle de muitas doenças, inclusive a aids, que tornará necessárias pesquisas que envolvam voluntários humanos. Isso pode ser confirmado pelos cerca de 7.500 novos casos de infecção pelo HIV diariamente ${ }^{12}$, mais de $90 \%$ deles em países em desenvolvimento. O mesmo ocorre com outros agravos, tais como tuberculose, hepatites virais, leishmaniose, malária e hanseníase. Entretanto, é inaceitável que tal urgência seja utilizada como justificativa para diminuir os padrões éticos dos ensaios clínicos ${ }^{3,13-16}$.
Se os pesquisadores são em geral pessoas privilegiadas, muitos dos voluntários estão entre as populações mais vulneráveis do mundo, vivendo em privação, que facilita a sua exploração, com pouca ou nenhuma voz nas decisões sobre onde e como a pesquisa deve ocorrer. A justificativa apresentada de que essas comunidades precisam com urgência de respostas a perguntas específicas da pesquisa em geral não é verdadeira, pois frequentemente os benefícios da pesquisa não Ihes serão acessíveis.

A necessidade urgente e real dessas comunidades é ter acesso a produtos pesquisados, desenvolvidos e muitas vezes já utilizados em outros lugares. Alega-se, sofisticamente, que há desigualdade em todos os lugares e que a pergunta específica da pesquisa só pode ser respondida em uma comunidade específica (muitas vezes miserável). Mesmo nas poucas situações em que esta especificidade poderia ser defendida, a vulnerabilidade dos participantes potenciais da pesquisa torna quase impossível conseguir consentimento realmente livre e esclarecido. Assim, nas situações como as listadas em documento da Organização Mundial da Saúde (OMS) relacionado aos cuidados e ética na pesquisa sobre tuberculose, apresentadas no Quadro 1, ao final, recomenda-se até mesmo que a pesquisa não seja realizada ${ }^{17}$. Nestes casos, há que se adequar às condições locais ou buscar regiões/comunidades onde possa ser executada, com o compromisso de que os resultados serão disponibilizados onde forem necessários.

Outra atual questão refere-se ao acesso aos serviços de saúde durante e após o ensaio. É mais que justo afirmar que não pode haver duplo padrão, todos devem ter acesso aos comprovadamente meIhores cuidados de saúde, mesmo quando existam limitações econômicas. A discussão econômica não pode ser utilizada para diminuir os requisitos éticos.

Estes aspectos relativos à proteção aos participantes em estudos clínicos revelam controvérsias em relação às normas éticas para a condução de pesquisas, como a Declaração de Helsinque (DH) ${ }^{18}$, que ao longo das últimas quatro décadas se tornou referência na proteção aos participantes nos ensaios. Entretanto, mudanças recentes na DH (2008) diminuíram a efetividade de sua proteção. Partindo do princípio de que vivemos num mundo desigual, qualquer mudança deveria visar que as obrigações éticas com os mais vulneráveis se tornem ainda mais rigorosas, bem como sua aplicação em todas as pesquisas envolvendo seres humanos, onde quer que sejam realizadas - e não subsumi-las.

Na versão 2000 da Declaração de Helsinque um dos pontos fundamentais de discussão estava 
nos itens 19 e 30. No primeiro, a pesquisa clínica só é justificada se houver razoável probabilidade de que as populações onde a pesquisa é realizada se beneficiem de seus resultados. Este item foi mantido na versão 2008. Entretanto, foi modificado o item 30, que garantia o acesso aos produtos da pesquisa, inclusive medicamentos, independentemente de seu local de realização. Definia, ainda, que como a participação como voluntário pressupõe equilíbrio entre ônus e benefícios não há qualquer justificativa para interromper o acesso, após a conclusão do estudo, ao que se mostrou benéfico durante a pesquisa. A modificação de 2008 facilita o seu não cumprimento, abrindo a possibilidade de que os benefícios a serem compartilhados não sejam o acesso às intervenções eficazes, mas a outros cuidados "apropriados", o que é vago.

Outra controvérsia frequente e também sofista é que não se pode esperar dos financiadores e pesquisadores que assumam o ônus, pois isto inibiria pesquisas importantes para o atendimento direto das necessidades de saúde dessas populações. Aceitar esse argumento é um protecionismo mal orientado em favor das agências e/ou indústrias farmacêuticas. Em geral, emprega-se o argumento dos altos custos para não manter tratamento para os voluntários após o final do experimento, sem avaliação sistemática destes custos, que provavelmente representam minúscula fração dos orçamentos usualmente multimilionários da maioria dos ensaios. $\mathrm{O}$ argumento de que, se implementadas, as provisões de acesso pós-ensaio (item 30, sem as modificações de 2008) comprometeriam pesquisas necessárias é da mesma forma falho. O Brasil é exemplo que confirma a falácia do argumento, pois o CNS/Conep manteve esta exigência de acesso e os ensaios clínicos aqui propostos não foram reduzidos, ao contrário, seguem crescentes.

\section{Precedente e concomitante - Tuskegee}

Para compreender estes pontos controversos, bem como a dinâmica que envolve os ensaios clínicos, é necessário voltar a 1932 e relembrar o que ocorreu em Tuskegee, Alabama. À época, o Serviço de Saúde Pública dos Estados Unidos da América (EUA) iniciou projeto para estudar a história natural da sífilis entre afro-americanos pobres, trabalhadores em fazendas de algodão. Este estudo, conhecido como a experiência de Tuskegee, revelou-se estarrecedor. Foram recrutados 399 homens, infectados pelo Treponema pallidum. Ainda que na primeira década de realização do estudo não houvesse tra- tamento adequado para a sífilis, a penicilina foi disponibilizada no início da década de 40 , tornando-se o tratamento padrão em torno de 1947. Entretanto, nenhum dos "voluntários" obteve permissão para receber este medicamento.

Como consequência, dos 399 participantes 28 morreram em decorrência da doença e 100, de complicações relacionadas; 40 esposas foram infectadas e houve pelo menos 19 casos de sífilis congênita. É inaceitável que a pesquisa tenha prosseguido mesmo após outras atrocidades cometidas durante a $2^{\mathrm{a}}$ Guerra Mundial terem sido condenadas pelo Tribunal de Nüremberg (1947). E que tenha continuado mesmo após a aprovação, em 1964, da primeira DH.

A experiência de Tuskegee foi publicada em várias revistas de prestígio, relatando a história natural da infecção, aparentemente sem despertar qualquer questionamento ético dos editores ou leitores. A exceção foi Peter Buxton, assistente social do Departamento de Saúde Pública dos EUA (PHS), que tomou conhecimento do experimento e empenhou-se em uma cruzada solitária para interrompê-lo. Sua postura desencadeou processo de avaliação ética interna, que concluiu por sancionar a continuidade do estudo. Discordando desta avaliação, Buxton relata a história para a imprensa em 1972. Jean Heller, repórter do The Washington Star, publica uma série de artigos com repercussão imediata nas televisões e outros jornais, inclusive em artigo de capa no The New York Times - o que leva, finalmente, à interrupção definitiva da experiência ${ }^{19}$. O governo estadunidense disponibilizou pequena indenização aos sobreviventes ou seus cônjuges e, 25 anos mais tarde (1997), o presidente Clinton reuniu sobreviventes e familiares dos falecidos na Casa Branca, desculpando-se publicamente:

O Governo dos Estados Unidos fez uma coisa errada, profunda e moralmente errada, que ultraja nosso comprometimento com a integridade e igualdade de todos os nossos cidadãos. Podemos acabar com o silêncio. Podemos parar de olhar para o outro lado. Podemos olhar em seus olhos e finalmente dizer, em nome do povo americano, que o Governo dos Estados Unidos fez uma coisa vergonhosa, e que eu sinto muito... O legado de Tuskegee teve forte e profundo alcance, prejudicando nosso progresso e dividindo nossa nação. Não podemos ser uma única América quando todo um segmento da nação desconfia da América. Um pedido de desculpas é o primeiro passo e nós o damos com o comprometimento de reconstruir a confiança perdida. Para começar, é preciso garantir que episódios como esse jamais venham 
a acontecer novamente. Precisamos nos empenhar mais para assegurar que as práticas de pesquisas médicas sejam sólidas e éticas, e que os pesquisadores trabalhem mais estreitamente ligados às comunidades ${ }^{20}$.

Em 1966, apenas alguns anos antes do cancelamento de Tuskegee, outro importante relato sobre a ética em pesquisas (ou a sua ausência) foi publicado por Henry Beecher ${ }^{21}$. Neste artigo, relata diversas pesquisas não éticas publicadas entre 1952 e 1965, em periódicos de prestígio, incluindo o New England Journal of Medicine (NEJM) e o Lancet. Descrevia abusos flagrantes, inclusive contra o princípio do respeito à pessoa, já que muitos participantes nem sabiam que participavam de um estudo e, portanto, foram expostos a situações em que havia risco de morte sem conhecimento.

Após as revelações de Buxton, a publicação de Beecher e o encerramento da experiência de Tuskegee em 1972, foram instaurados diversos inquéritos pelo poder legislativo norte-americano para lidar com a improbidade ética em pesquisas. Em 1974, foi criada a Comissão Nacional para a Proteção de Sujeitos Humanos em Pesquisas Biomédicas e Comportamentais, para identificar os princípios éticos básicos que deveriam fundamentar a conduta em pesquisas envolvendo seres humanos e desenvolver diretrizes para assegurar que sejam conduzidas em conformidade com aqueles princípios. Incluíam-se nessas considerações: os limites entre a pesquisa biomédica e comportamental e a prática aceita e rotineira da medicina; o papel da avaliação dos critérios risco-benefício para determinar a adequação de pesquisas envolvendo seres humanos; as diretrizes apropriadas para a seleção de participantes em pesquisas; e a natureza e definição do consentimento esclarecido nos diversos ambientes de pesquisa.

Em 1979, a Comissão publicou "Princípios éticos e diretrizes para a proteção de sujeitos humanos em pesquisas", conhecido como Relatório Belmonte ${ }^{22}$, que estabeleceu três princípios éticos para a pesquisa com seres humanos: a autonomia dos participantes sempre deve ser respeitada; a beneficência (fazer o bem) deve fundamentar a conduta em todas as pesquisas com seres humanos; a justiça deve sempre prevalecer na pesquisa com seres humanos. Um quarto princípio foi posteriormente acrescentado: o princípio da não maleficência (não prejudicar).

As normas básicas para a proteção de seres humanos em pesquisas financiadas ou realizadas pelo Departamento de Saúde, Educação e Previdên- cia Social foram publicadas em 1974, com a aprovação do Ato de Pesquisa Nacional (Lei 93-348) que criou a comissão. As normas para a proteção de sujeitos humanos, baseadas principalmente no Relatório Belmonte, foram expandidas no final dos anos 70. Vale observar que somente em 1991 outros 14 departamentos e agências federais envolvidos com o tema adotaram conjunto uniforme de regras para esta proteção, idêntico ao do Código das Regulamentações Federais (CFR) 45, parte 46, subparte A. Esse conjunto de regras constitui a política federal norte-americana para a proteção de sujeitos humanos, conhecida como Regra Comum (Common Rule). Os principais requisitos incluem: garantia de conformidade pelas instituições de pesquisa; obtenção e documentação do consentimento esclarecido pelos pesquisadores; estabelecimento de Comissão de Revisão Institucional (Institutional Review Board), que equivale aos comitês de ética em pesquisa brasileiros - com especificação de membros, funcionamento, funções, operações, revisão de pesquisas e manutenção de registros; e proteções adicionais para populações vulneráveis específicas - mulheres grávidas, prisioneiros e crianças.

\section{A experiência da Guatemala}

Em 2010, Susan Reverby, da Faculdade de Wesllesley ${ }^{9}$, revelou que na década de 40 o Departamento de Saúde Pública deliberadamente inoculou prisioneiros e profissionais do sexo na Guatemala com doenças sexualmente transmissíveis, principalmente o T. pallidum. Incluíam-se nos objetivos do estudo descobrir se o uso profilático da penicilina poderia evitar a infecção e avaliar sua eficácia. 0 consentimento não foi obtido e muitos recrutados não tiveram acesso a tratamento adequado.

Essa descoberta provocou reações entre especialistas em ética de todo o mundo e o atual governo estadunidense divulgou pedido de desculpas à Guatemala: Embora esses eventos tenham ocorrido há mais de 60 anos, é revoltante que pesquisa tão repreensivel possa ter ocorrido sob o pretexto da saúde pública (...) O estudo é um triste lembrete de que salvaguardas adequadas para os seres humanos não existiam há meio século ${ }^{10}$. Acrescentaram que as atuais diretrizes de pesquisa médica em humanos, financiadas pelos EUA, proíbem esse estarrecedor tipo de violação:

No espírito de comprometimento com a pesquisa ética, estamos lançando investigação rigorosa sobre todos os aspectos desse caso. Além disso, por meio 
da Comissão Presidencial para o Estudo de Questões Bioéticas, estamos reunindo um grupo de especialistas internacionais para rever estes métodos e para buscar os mais eficazes para garantir que todas as pesquisas médicas com seres humanos conduzidas atualmente no mundo todo atendam padrões éticos rigorosos ${ }^{10}$.

Foi então criado, em março de 2011, o Painel Internacional Presidencial dos EUA, com quatro membros da Comissão Presidencial para o Estudo de Questões Bioéticas e 13 especialistas internacionais. O presidente Obama orientou a Comissão a supervisionar investigação para apuração completa dos fatos e a realizar revisão das proteções para pessoas que se submetem a experimentos, para determinar se as normas federais e os padrões internacionais resguardam a saúde e o bem-estar dos participantes contra danos ou tratamentos não éticos, em estudos científicos financiados pelo Governo norte-americano nacional e internacionalmente ${ }^{23}$. Este Painel se reuniu presencialmente três vezes, sendo a última em Washington, em julho de $2011^{11}$.

\section{Como os voluntários eram infectados}

No início, prostitutas infectadas por sífilis foram recrutadas para ter relações sexuais com prisioneiros. Como a taxa de transmissão foi baixa, os pesquisadores prepararam amostras infectadas para inocular os participantes, mediante muitos métodos. As espiroquetas vivas no inóculo, preparado a partir de raspagens de cancros dos prisioneiros ou de militares infectados, sobreviviam por períodos muito curtos fora do corpo, levando à utilização de material com espiroquetas mortas pelo calor. Este material era transferido para os sujeitos por abrasão da pele, injeção ou inoculação pela uretra.

Há outros exemplos dos inaceitáveis procedimentos utilizados: nas prisioneiras o inóculo era introduzido por escarificação nos braços, rosto ou boca ${ }^{23}$. Nos homens, a inoculação era geralmente mais direta. Foram escolhidos homens com prepúcios no mínimo moderadamente longos [para manter as membranas mucosas úmidas] e que podiam ficar sentados ou em pé calmamente em um lugar durante várias horas. O médico segurava o pênis do participante, puxava o prepúcio e escarificava o pênis com uma agulha hipodérmica; em seguida, introduzia um chumaço de algodão (ou gaze pequena) e gotejava a emulsão de sífilis, que ficava em contato com a pele lacerada do pênis durante pelo menos uma hora, algumas vezes, até duas horas ${ }^{23}$.
Outras técnicas foram testadas, tais como a injeção intravenosa, a ingestão de suspensão em água ou a colocação do inóculo no colo do útero das prostitutas antes da relação sexual. Diferentes tipos de produtos profiláticos químicos foram fornecidos a alguns dos participantes e nenhum para o grupo controle. Antes do recrutamento, certificaram-se que não estavam infectados ou que não haviam recebido medicamento para a sífilis.

Centenas de homens e mulheres foram envolvidos, muitos fotografados e suas fotos encontradas nos arquivos. Alguns participaram em múltiplas experiências. Os números totais apurados pela Comissão Presidencial foram 696 pessoas expostas à sífilis, 722 à gonorreia e 142 ao cancroide ${ }^{23}$.

\section{Engodo}

O principal pesquisador da experiência da Guatemala também desempenhou importante papel em Tuskegee. John Cutler, cirurgião que supervisionou o projeto guatemalteco, tornou-se cirurgião geral assistente em 1958 e participou de Tuskegee na década seguinte. Em sua correspondência com médicos interessados em infecções sexualmente transmissíveis, admitiu que apenas algumas pessoas (não os participantes, nem seus colegas do PHS e da comunidade científica) tinham conhecimento do procedimento completo: estamos explicando para os pacientes e outros envolvidos, com algumas poucas exceções, que se trata de um novo tratamento utilizando soro seguido de penicilina. Às vezes este tipo de conversa ambígua me deixa apreensivo ${ }^{23}$. Em carta, comenta: algumas palavras ditas para a pessoa errada aqui, ou mesmo em casa, podem destruir o projeto ou parte dele ${ }^{23}$.

\section{Participação de funcionários guatemaltecos}

Um projeto desta envergadura não poderia ter acontecido sem a colaboração de muitas pessoas, inclusive de autoridades e médicos guatemaltecos, sujeitos, portanto, a diversos conflitos de interesse ${ }^{24}$. Entre esses interesses estava a possibilidade de progredir na carreira com a associação com pesquisadores e instituições norte-americanas renomadas. 0 projeto trazia ainda a implementação de infraestrutura laboratorial, a possibilidade de acesso a exames e de maior acesso do país à penicilina.

Adicionalmente, Cutler acrescentou o fornecimento de medicamentos para a malária em troca do 
direito de continuar a fazer exames de sangue. Desde a descoberta da penicilina o PHS estava enfrentando restrições orçamentárias nos trabalhos sobre doenças venéreas, dificultando justificar o projeto da Guatemala. Após diversas cartas, Cutler prometeu ser cuidadoso: utilizaremos nosso estoque com moderação de modo a tê-lo sempre disponível para uso em programas de demonstração e para construir uma relação de boa vontade ${ }^{23}$. Também destacou: com a oportunidade que se oferece aqui para estudar a sifilis de uma perspectiva puramente científica, da mesma forma que Chesney a estuda com coelhos (...) seria possível justificar os projetos no caso de impossibilidade de resolução do programa profilático ${ }^{23}$.

Chama a atenção uma pergunta específica endereçada ao Painel Internacional pelo presidente Obama, sobre se haveria, hoje, o risco de experimentos similares. Embora se considere que as normas federais e os padrões internacionais protegem de maneira adequada a saúde e o bem-estar dos participantes em estudos científicos ${ }^{23}$, sendo muito improvável que um projeto como o da Guatemala seja permitido atualmente, deve-se atentar para o fato de que há outras maneiras, sutis e também não éticas em ensaios clínicos, tanto nos Estados Unidos como em outros países, devido às desigualdades de poder (vulnerabilidade) e falta de aceitação (ou reconhecimento) de uma diretriz ética internacional representativa, tal como a Declaração Universal sobre Bioética e Direitos Humanos da Unesco ${ }^{25}$, que sequer foi mencionada no relatório final do Painel Internacional ${ }^{26}$.

Assim, não é possível concluir que a saúde e o bem-estar de participantes estejam de fato protegidos de maneira adequada em todos os estudos financiados pelo governo federal americano e ainda menos em projetos apoiados e desenvolvidos pela indústria farmacêutica. Uma maneira de lidar com essa questão, pelo menos para começar, é garantir que normas éticas universalmente reconhecidas sejam adotadas, conhecidas por todos e que uma estrutura ética independente realmente funcione em cada país onde a pesquisa está sendo proposta.

\section{Soluções possíveis}

O que deve ser discutido e decidido é como garantir o papel das diretrizes ou melhor, de uma diretriz universal, como a citada Declaração da Unesco, para lidar de maneira efetiva com questões de relevância (ou capacidade de resposta), justiça e as disparidades. E tão importante quanto, a apropriação pelo país das suas necessidades de pesquisas, por meio da comunidade, pesquisadores locais, comitês de ética em pesquisa realmente independentes e autoridades da saúde. São questões realmente polêmicas que, apesar de discutidas, não são enfrentadas com rigor. Não há consenso nos diversos países e também internacionalmente sobre as questões de relevância/capacidade de resposta, justiça, acesso aos produtos da pesquisa, uso de placebo, riscos de exploração com relação ao desempenho de pesquisas questionáveis com voluntários/comunidades/pesquisadores/países vulneráveis.

Contudo, cumpre dar crédito ao presidente dos EUA por sua decisão de revelar mais uma vez, à semelhança do citado pedido público de desculpas do presidente Clinton por Tuskegee, outra parte deplorável da história daquele país. Além disso, em março de 2013 a Comissão de Bioética da Presidência dos EUA disponibilizou pela primeira vez os dados referentes a 55 mil ensaios clínicos financiados pelo governo federal no período de 2006 a $2010^{27}$. Esta medida reforça o que preconiza o item 30 da Declaração de Helsinque ${ }^{18}$, definindo a obrigação da publicação ou publicização dos resultados positivos ou negativos dos ensaios clínicos, incluindo fonte de financiamento, afiliação institucional e possíveis conflitos de interesse. Evidentemente, revelar não é o suficiente, mas pode e deve ser utilizado como oportunidade para ampliar a discussão ética e meIhorar o delineamento das questões de autonomia, relevância, igualdade e riscos de exploração dos vulneráveis.

Riscos de situações similares na estrutura ética em pesquisa no Brasil

Como o Brasil protege os voluntários dos projetos de pesquisa? A estrutura ética em pesquisa brasileira tem como base a Resolução CNS 196/96, que criou a Conep. Nesta resolução estão claramente estabelecidos os passos necessários para criar e registrar comitês de ética em pesquisa (CEP) locais; definida a estruturação de um projeto, com todas as salvaguardas para a proteção de voluntários humanos, incluindo, mas não se limitando, o processo do consentimento livre e esclarecido ${ }^{28}$.

A Resolução CNS 196/96 está sendo atualizada (2013) e há diversas resoluções complementares para situações/populações específicas, com maior risco de vulnerabilidade. Com este conjunto de normas, propostas de pesquisa similares à de Tuskegee 
ou ao estudo da sífilis na Guatemala não seriam permitidas no Brasil. Entretanto, é inegável a pressão por normas mais flexíveis, que podem enfraquecer os requisitos éticos, utilizando os já conhecidos sofísticos argumentos da urgência e de que não é realista ser mais rígido que o resto do mundo, além do risco inerente à vulnerabilidade dos voluntários em pesquisas brasileiras, geralmente recrutados nos serviços públicos muitas vezes sobrecarregados. Estes, ao serem recrutados, passam a ter acesso privilegiado a cuidados de saúde, que não tinham antes, dificultando inclusive o consentimento realmente livre.

As modificações na Declaração de Helsinque (2008) exemplificam como uma regulamentação internacional relacionada à ética em pesquisa pode não corresponder aos interesses dos países em desenvolvimento, devido à influência das agências de fomento/financiadoras e da indústria farmacêutica dos países centrais. Este foi o caso desta última versão da $\mathrm{DH}$, que poderia ter influenciado negativamente as diretrizes de pesquisa brasileiras, mas que foi prontamente combatida pela posição soberana e inequívoca do CNS, com a aprovação da Resolução 404/08 ${ }^{29}$, e do Conselho Federal de Medicina (CFM), com a Resolução é 1.885/08 ${ }^{30}$, ambas mantendo as conquistas da versão 2000 da DH.

Estas decisões do CNS e do CFM definem que a autonomia, o processo de consentimento verdadeiramente esclarecido e o acesso ao cuidado e tratamento durante e após os ensaios clínicos são direitos de todos os voluntários em ensaios de pesquisa. Todos esses requisitos devem integrar uma perspectiva mais ampla e necessária, que é a expansão do direito de acesso aos benefícios da pesquisa na saúde pública. Como visto, as últimas décadas presenciaram impressionante expansão de ensaios clínicos originados em países desenvolvidos, em geral conduzidos em países em desenvolvimento ${ }^{31}$.

O aumento no número de ensaios ocorre, entre outras razões, também pela necessidade de conformidade com as regras para a realização de ensaios clínicos randomizados de grande escala. 0 estabelecimento de ensaios clínicos fase III (avaliação da eficácia), com milhares de voluntários, e a globalização da indústria farmacêutica contribuíram para a expansão dos ensaios fora do país de origem da indústria. Talvez ainda mais importante, a facilidade de recrutamento de voluntários em ambientes mais pobres, muitas vezes com estrutura ética de pesquisa menos organizada, com alta incidência da doença e indivíduos menos exigentes, que muitas vezes veem a oportunidade de participação como o único meio de obter atendimento médico. Paralela- mente, normas mais rígidas nos países desenvolvidos, onde os direitos dos voluntários são protegidos pelo menos na lei, fazem parte do mesmo quadro que facilita a migração dos ensaios clínicos.

\section{Duplo padrão em ensaios clínicos}

A migração de ensaios randomizados controlados para o Terceiro Mundo traz o risco de que se tente diminuir as exigências éticas internacionais. Não há aqui a intenção de demonizar a indústria farmacêutica, que tem seu papel no processo de desenvolvimento de produtos necessários, qualificando-a como única condutora de ensaios antiéticos. Entretanto, existem diversos relatos de ensaios não éticos, em época recente, para o desenvolvimento de medicamentos ou avaliação de métodos preventivos patrocinados pela indústria farmacêutica ${ }^{32}$. Cabe detalhar o catalisador específico e recente de discussões acirradas sobre requisitos éticos em ensaios clínicos.

Em 1997, enérgico editorial de Marcia Angell ${ }^{33}$ e artigo de Wolfe e Lurie ${ }^{34}$, publicados na mesma edição do NEJM, criticaram a ética dos estudos financiados pelo Instituto Nacional de Saúde (NIH) dos EUA sobre a prevenção da transmissão vertical do HIV, realizados em países da África, República Dominicana e Tailândia, no final da década de 80. Pelo estudo ACTG 076 sabia-se que zidovudina por via oral durante a gravidez, endovenosa durante o parto e oralmente para o neonato reduzia substancialmente o risco de transmissão. Os ensaios nestes países utilizaram esquema mais curto do AZT, eliminando o uso intravenoso. Além disso, em vez de ser comparado com o protocolo do ACTG 076, o ensaio utilizou placebo como controle. Angell, editora-chefe do NEJM, questionou a metodologia empregada remetendo-a àquela de Tuskegee.

O editorial e o artigo provocaram discussões acirradas que, por sua vez, traduziram-se em forte pressão para alterar a Declaração de Helsinque (versão 1996), o principal e mais respeitado conjunto de normas éticas para pesquisa com seres humanos. O objetivo era modificar dois artigos, precisamente aqueles que tratavam do acesso ao melhor tratamento médico comprovado a todos os voluntários, sem considerar condição econômica ou país de origem; e as restrições ao uso de placebo quando existe tratamento eficaz. A Assembleia Geral da Associação Médica Mundial, em Edimburgo (2000), manteve as restrições ao uso de placebos (item 29) e acrescentou a exigência de acesso pós-estudo ao 
produto que se mostrar eficaz (item 30). As pressões, tanto da indústria farmacêutica quanto das agências americanas, foram intensas o suficiente para que estas exigências ficassem muito menos rígidas na versão 2008 da $\mathrm{DH}$, como discutido.

Por consequência, abria-se a possibilidade de que os ensaios se esquivassem das exigências de tratar os voluntários com igual respeito e garantias, independentemente da sua origem e poder econômico, criando a possibilidade de duplo padrão nas pesquisas clínicas. As pressões também contaminaram outros importantes documentos, como as Considerações éticas sobre ensaios com vacinas anti-HIV (Unaids, 2000) ${ }^{35}$ e as Diretrizes Éticas Internacionais sobre Pesquisas Biomédicas Envolvendo Seres Humanos (Cioms, 2002) ${ }^{36}$. Nos dois documentos, os itens relacionados ao acesso a atendimento médico e uso de placebos são longos, de complexo entendimento e possibilitam tratar voluntários de países em desenvolvimento de maneiras diversas, isto é, com menos direitos que os do mundo industrializado.

Acrescente-se a isso o surgimento, nos países industrializados, de outros documentos focados nas pesquisas em países em desenvolvimento. Um exemplo é o documento da Comissão Nacional de Assessoramento Bioético dos Estados Unidos (US National Bioethics Advisory Commission - 2001) ${ }^{37}$. A este, seguiu-se um documento da Administração de Drogas e Alimentos (FDA) ${ }^{38}$, aprovado em 2008, definindo que projetos de pesquisa clínica realizados fora dos EUA e não conduzidos sob um pedido de investigação de nova droga (IND) não mais precisam estar acordes com as normas da Declaração de Helsinque, mas apenas com as Boas práticas clínicas da Conferência Internacional de Harmonização (ICH). Vale lembrar que o objetivo precípuo das diretrizes da $\mathrm{ICH}^{39}$ é unificar os procedimentos europeus, norte-americanos e japoneses para facilitar a aceitação mútua de dados clínicos pelas agências reguladoras destes países. Trata-se - mais uma vez - dos mesmos riscos de padrões duplos ${ }^{40,41}$.

\section{Contra-ataque}

Nos primeiros anos do século XXI, mais precisamente em 2005, uma vitória foi conquistada com a promulgação, por aclamação dos Estados-mem-

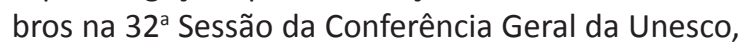
da Declaração Universal sobre Bioética e Direitos Humanos. Trata-se de marco real e efetivo na busca pela justiça e igualdade para todos. Nas Provisões Gerais, o item " $f$ " do artigo 2 define dentre seus objetivos: promover o acesso igualitário aos desenvolvimentos médicos, científicos e tecnológicos, bem como encorajar o fluxo e o compartilhamento mais rápido possivel do conhecimento relativo a esses desenvolvimentos e de seus benefícios, com especial atenção às necessidades dos países em desenvolvimento ${ }^{26}$.

Os objetivos da Declaração, que estão em completa consonância com o escopo deste artigo, incluem a universalidade dos princípios e também dos procedimentos, enfatizando a necessidade de respeito à dignidade humana e de proteção dos direitos humanos e, mais importante, a obrigatoriedade do acesso igualitário aos desenvolvimentos médicos, científicos e tecnológicos, o compartilhamento dos benefícios alcançados e atenção especial às necessidades dos países em desenvolvimento. $A$ Declaração reforça ainda a posição emanada pelo CNS do Brasil em relação à proteção efetiva aos voluntários de pesquisa, contra qualquer possibilidade de duplo padrão nos estudos.

Documento similar - Considerações éticas para ensaios biomédicos para a prevenção do HIV ${ }^{42}$ - foi publicado em 2007 pela OMS e Joint United Nations Programme on HIV/Aids (Unaids). Substituindo e ampliando o escopo da diretriz Pesquisa de vacinas preventivas para o HIV-Unaids 2000 , inclui recomendação explícita e direta de que os participantes infectados pelo HIV durante a condução do ensaio biomédico para a prevenção do HIV devem receber acesso a regimes de tratamento que sejam reconhecidos como excelentes em nível internacional (Diretriz 14). Em 2010, a OMS publicou diretriz sobre a ética para a prevenção, cuidado e controle da tuberculose ${ }^{17}$, anteriormente citada, que segue a mesma linha do documento da OMS/Unaids de 2007, incluindo a tabela que lista as circunstâncias nas quais os ensaios clínicos não devem ser realizados (Quadro 1).

\section{Acesso a serviços de saúde adequados para todos}

Toda a discussão sobre o direito dos voluntários de ensaios clínicos ao acesso igualitário a cuidados médicos decentes, independentemente de sua origem econômica ou do país onde a pesquisa está sendo conduzida, pode ser hoje considerada ultrapassada. Isto porque, ao longo deste período (1999-2008), as discussões sobre o acesso extrapolaram os ambientes controlados dos ensaios clínicos para uma expectativa muito mais ampla de acesso aos produtos desenvolvidos para todos que deles 
precisam. O cerne da questão não está no ensaio clínico controlado e em geral financiado por indústrias multimilionárias, mas na tarefa mais importante, mais difícil e menos visível que consiste em aplicar os resultados na saúde pública, em ambientes de recursos limitados.

Há exemplos neste sentido relacionados coincidentemente à epidemia da aids: a Iniciativa 3 por 5 (3 by 5) da OMS/Unaids em 2003, propondo que as drogas para o tratamento do HIV sejam de fato disponibilizadas em todos os países em desenvolvimento; e em 2011, aprovada na reunião de alto nível da ONU em Nova lorque, a Declaração Política sobre HIV/Aids, que entre seus objetivos traz a disponibilização de tratamento antirretroviral para 15 milhões de pessoas até 2015 (15 by 15) ${ }^{43}$. Outro exemplo foi a aprovação e implementação, no Brasil, da Lei 9.313/96, que estabeleceu acesso universal pelo Sistema Único de Saúde (SUS) aos antirretrovirais para todos os pacientes que deles necessitassem, sem custo adicional.

Essa decisão ajudou a reduzir as taxas de mortalidade, morbidade, hospitalizações, ausências no trabalho e novas infecções, além de se revelar válida também do ponto de vista econômico ${ }^{44}$. Entre 1996 e 2002, o investimento total com medicamentos ultrapassou US\$ 1,6 bilhão, com incalculável impacto social. Houve, ainda, significativo impacto econômico, com economia estimada em outros US\$ 2 bilhões em decorrência da redução nas hospitalizações, cuidados ambulatoriais, despesas com previdência e mortalidade.

\section{Determinantes sociais e as relações de poder}

O termo empoderamento tem sido repetido ad nauseam como forma de "capacitar" indivíduos com relação às suas necessidades e expectativas. Infelizmente, em geral não passa de figura de retórica, vez que o poder nunca é dado e muito raramente compartilhado. Na maioria das vezes, o uso do termo corresponde a uma forma iluminista de fornecer de maneira marginal um pouco para os necessitados, objetivando acalmar suas reivindicações. É comum que esses indivíduos/países com dificuldades econômicas enalteçam os "doadores" por ajudá-los a obter algo que é de fato seu direito. Essa atitude pode perpetuar a dependência: nasce novo tipo de colonialismo (entreguem os anéis, salvem os dedos e perpetuem a disparidade e a dependência).

O termo "empoderamento", do inglês empowerment, deveria ser substituído por emancipação, no sentido de Paulo Freire, que em sua abrangente obra sobre a educação para a liberdade empregou a palavra com sentido amplo, de libertação e autonomia, exatamente como deve ser utilizada quando discutimos cidadania, direitos ou a luta contra disparidades ${ }^{45}$. Marx também indicou que a emancipação humana só é realizada quando o homem reconhece e organiza as suas próprias forças como forças sociais, deixando, pois, de separar de si a força social sob a forma de força política ${ }^{46}$. Assim, a emancipação não acontecerá por eventualidade, por concessão, mas será uma conquista efetivada pela práxis humana, que demanda luta ininterrupta: A libertação, por isto, é um parto (...) O homem que nasce deste parto é um homem novo que só é viável na e pela superação da contradição opressores-oprimidos, que é a libertação de todos ${ }^{47}$.

\section{Considerações finais}

Ensaios com novos medicamentos ou vacinas são necessários e devem ser realizados em locais onde requisitos éticos e avaliações independentes estejam disponíveis, onde a exploração possa ser evitada, quando a pesquisa proposta aborda problemas de saúde locais e quando há garantias de acesso ao melhor método diagnóstico e terapêutico comprovado.

Uma estrutura bem definida e adequada de ética em pesquisa é fundamental e o envolvimento de cientistas, ativistas e de toda a sociedade é essencial para garantir que requisitos éticos acordados internacionalmente sejam utilizados corretamente em todo o mundo, evitando o risco do duplo padrão; que pessoas recebam tratamento diferente e geralmente pior em função de sua origem, etnia ou situação econômica.

Em relação às pesquisas envolvendo seres humanos, há procedimentos capazes de reduzir de maneira parcial esses problemas, que incluem diretriz internacional sobre ética em pesquisa, aprovada e universalmente aplicável; estabelecimento de comitês de ética em pesquisas locais/nacionais soberanos e independentes, acompanhados da divulgação pública das propostas e resultados das pesquisas. Com todos esses instrumentos será possível impedir que se repitam não só experimentos como os de Tuskegee, Guatemala e os ensaios com AZT para mães infectadas pelo HIV, mas também muitos outros igualmente não éticos. Se, ao final da pesquisa, o produto se mostrou eficaz, deve haver pressão internacional para disponibilizá-lo e tornálo acessível financeiramente para uso em outros 
países. Existe urgência, portanto, não apenas para pesquisar métodos preventivos melhores ou drogas e vacinas mais eficazes, mas principalmente para disponibilizá-los para todos.

Contudo, a tarefa mais difícil, aquela da qual todos temos de fazer parte, é o desafio de lutar de fato contra as disparidades que separam os poucos ricos, tanto em países desenvolvidos como em desenvolvimento, dos milhões de destituídos que ainda não têm voz nem direitos. Infelizmente, as disparidades de saúde não serão solucionadas apenas por meio dos padrões e diretrizes que regulamentam as pesquisas e os pesquisadores, ou mesmo pelo tratamento igualitário a todos nos estudos envolvendo seres humanos.

Cumpre ressaltar importante passo adotado consensualmente pelos países na $67^{\mathrm{a}}$ Assembleia
Geral das Nações Unidas, em 2012, para transição dos sistemas nacionais de cuidados de saúde em direção à cobertura universal em saúde ${ }^{48}$. Tucídides ${ }^{49}$ afirmou que a justiça só será alcançada quando os que não são injustiçados sentirem-se tão indignados quanto aqueles que o são. Atrevo-me a dizer que a justiça só prevalecerá quando aqueles afetados e indignados pela injustiça forem capazes de se emancipar para a luta por seus direitos.

Garantir que a igualdade e os direitos sejam respeitados nas pesquisas que envolvem os seres humanos em qualquer lugar do mundo é significativo passo para reverter a injustiça atual na alocação de recursos para a saúde e pode contribuir para a emancipação dos voluntários, pesquisadores e sociedade, para que exerçam seus direitos enquanto cidadãos e a capacidade de lutar por eles.

\section{Referências}

1. Angell M. Investigators' responsibilities for human subjects in developing countries. N England J Med. 2000;342(13):967-8.

2. Bayer R, Gostin L. Legal and ethical issues in Aids. In: Gotlieb MS. Current topics in Aids. New York: John Wiley and Sons; 1989. p. 263-86.

3. Brennan TA. Proposed revision of the Declaration of Helsinki: will they weaken the ethical principles underlying human research? Bull Med Ethics. 1999;(150):24-8.

4. Greco DB. Science, ethics and future of research into maternal-infant transmission of HIV-1. Lancet. 1999;353(9167):1878.

5. Benatar SR. Global disparities in health and human rights. Am J Public Health. 1998;88(2):295300.

6. Shuster E. Fifty years later: the significance of the Nuremberg Code. N England J Med. 1997;337(20):1436-40.

7. Fairchild AL, Bayer R. Uses and abuses of Tuskegee. Science. 1999;284(5416):919-21.

8. Jones JH. Bad blood: the Tuskegee syphilis experiment. New York: Free Press; 1993.

9. Reverby SM. "Normal Exposure" and inoculation: a PHS "Tuskegee" doctor in Guatemala, 194648. Journal of Policy History. Jan 2011;Special Issue on Human Subjects.

10. U.S. Department of State. Joint statement by Secretaries Clinton and Sebelius on a 19461948 study. [internet]. 1 oct. 2010 [acesso oct. 2012]. Disponível: www.state.gov/secretary/ $\mathrm{rm} / 2010 / 10 / 148464 . \mathrm{htm}$

11. U.S. Presidential Commission for the Study of Bioethical Issues. Names International Research Panel: takes critical step in fulfilling President Obama's charge stemming from 1940s sexually transmitted disease experiments in Guatemala. [internet]. [acesso oct. 2012]. Disponível: http:// bioethics.gov/cms/node/13

12. United Nations Programme on HIV/Aids. World Aids Day Report 2011. [internet]. 2011 [acesso oct. 2012]. Disponível: http://www.unaids.org/en/media/unaids/contentassets/documents/ unaidspublication/2011/jc2216_worldaidsday_report_2011_en.pdf

13. Greco DB. A cure at any cost? New Scientist. 1 July 2000;(2245):42-3.

14. Greco DB. Revising the Declaration of Helsinki: ethics vs. economics or the fallacy of urgency. Can HIV/Aids Policy Law Rev. 2000;5(4):98-101.

15. Dismantling the Helsinki Declaration (Editorial). CMAJ. 2003;10:169.

16. Levine RJ. The need to revise the Declaration of Helsinki. Bull Med Ethics. 1999;(150):29-33

17. World Health Organization. Guidance on ethics of tuberculosis prevention, care and control. Geneva: WHO; 2010. 29 p.

18. World Medical Association. Declaration of Helsinki. Ethical Principles for Medical Research Involving Human Subjects. [internet]. Tokyo: WMA; 2004 [acesso oct. 2012]. Disponível: http:// www.wma.net/en/30publications/10policies/b3/index.html

19. Jones J. The Tuskegee syphilis experiment. In: Emmanuel EJ, Grady C, Crouch RA, Lie RK, Miller FG, Wendler D, editors. The Oxford textbook of clinical research ethics. Oxford: Oxford University Press; 2008. p. 86-91. 
20. Clinton B. Remarks by the President in apology for study done in Tuskegee. [internet]. Washington: The White House; 16 may 1997 [acesso oct. 2012]. Disponível: clinton4.nara.gov/New/Remarks/ Fri/19970516-898.html

21. Beecher HK. Ethics and clinical research. New England J Med. 1966;274(24):1354-60.

22. The National Commission for the Protection of Human Subjects of Biomedical and Behavioral Research. Belmont Report: ethical principles and guidelines for the protection of human subjects of research, 18 april 1979. [internet]. Bethesda: Office of Human Suject Research; [acesso oct. 2012]. Disponível: http://ohsr.od.nih.gov/guidelines/belmont.html

23. U.S. Presidential Commission for the Study of Bioethical Issues. "Ethically impossible" STD research in Guatemala from 1946 to 1948. [internet]. Washington; 2011 [acesso oct. 2012]. Disponível: http://bioethics.gov/cms/sites/default/files/Ethically-Impossible_PCSBI.pdf

24. Greco DB, Diniz NM. Conflicts of interest in research involving human beings. J Int Bioetique. 2008;19:143-54.

25. United Nations Educational, Scientific and Cultural Organizations. Universal Declaration on Bioethics and Human Rights. [internet]. Paris: Unesco; 2005 [acesso oct. 2012]. Disponível: http://unesdoc.unesco.org/images/0014/001461/146180E.pdf

26. U.S. Presidential Commission for the Study of Bioethical Issues. Research across borders: proceedings of the International Research Panel of the Presidential Commission for the Study of Bioethical Issues. [internet]. [acesso oct. 2012]. Disponível: http://bioethics.gov/cms/node/346

27. U.S. Presidential Commission for the Study of Bioethics Issues. Bioethics Commission posts data on federal supported research involving human volunteers: one of a kind dataset from 2006-2010 is example of information government could maintain. [internet]. Dec. 2011 [acesso oct. 2012]. Disponível: http://bioethics.gov/cms/node/834

28. Conselho Nacional de Saúde. Resolução n 196 , de 10 de outubro de 1996. [internet]. Aprova as diretrizes e normas regulamentadoras de pesquisas envolvendo seres humanos. 1996 [acesso out. 2012]. Disponível: http://conselho.saude.gov.br/resolucoes/1996/Reso196.doc

29. Conselho Nacional de Saúde. Resolução $n^{\circ} 404$, de 1 de agosto de 2008. [internet]. Propõe a retirada das notas de esclarecimento dos itens relacionados com cuidados de saúde a serem disponibilizados aos voluntários e ao uso de placebo. 2008 [acesso out. 2012]. Disponível: conselho.saude.gov.br/resolucoes/2008/reso_404.doc

30. Conselho Federal de Medicina. Resolução $n^{\circ} 1.885$, de 23 de outubro de 2008. [internet]. É vedado ao médico participar de pesquisa envolvendo seres humanos utilizando placebo, quando houver tratamento disponível eficaz já conhecido. 2008 [acesso out. 2012]. Disponível: http:// www.portalmedico.org.br/resolucoes/CFM/2008/1885_2008.htm

31. Macklin R. Double standards in medical research in developing countries. Cambridge: Cambridge University Press; 2004.

32. Lavery JV, Grady C, Wahl ER, Emanuel EJ, editors. Ethical issues in international biomedical research: a case book. Londres: Oxford University Press; 2007.

33. Angell M. The ethics of clinical research in the third world. N Engl J Med. 1997;337: 847-9.

34. Lurie P, Wolfe SM. Unethical trials of interventions to reduce perinatal transmission of HIV in developing countries. N England J Med. 1997;337:1003-5.

35. United Nations Programme on HIV/Aids. Ethical considerations in HIV preventive vaccine research. [internet]. Genebra: WHO/Unaids; 2000 [acesso oct. 2012]. Disponível: http://data.unaids.org/ publications/IRC-pub01/jc072-ethicalcons_en.pdf

36. Council for International Organizations of Medical Sciences. International ethical guidelines for biomedical research involving human subjects. [internet]. Geneva: Cioms; 2002 [acesso oct. 2012]. Disponível: http://www.cioms.ch/publications/guidelines/guideline_nov_2002.blurb. html

37. National Bioethics Advisory Commission. Ethical and policy issues in international research: clinical trials in developing countries. Bethesda, Maryland: NBAC; 2001. Volume 1: report and recommendations of the National Bioethics Advisory Commission.

38. U.S. Department of Health and Human Services. Food and Drug Administration. Code of Federal Regulation. [internet]. 2008 [acesso oct. 2012]. Disponível: http://www.gpo.gov/fdsys/pkg/CFR2003-title21-vol5/pdf/CFR-2003-title21-vol5-part312.pdf

39. International Conference on Harmonisation of Technical Requirements for Registration of Pharmaceuticals for Human Use. Efficacy guidelines. [internet]. 2012 [acesso oct. 2012]. Disponível: http://www.ich.org/products/guidelines/efficacy/article/efficacy-guidelines.html

40. Lurie P, Greco DB. US exceptionalism comes to research ethics. Lancet. 2006;26:1117-9.

41. "Trials on trial: the FDA should rethink its rejection of the Declaration of Helsinki" (Editorial). Nature. 2008; 453(7194): 427-8.

42. World Health Organization. Ethical considerations in biomedical HIV prevention trials. Geneva: WHO/Unaids; 2007.

43. Nações Unidas. Declaração Política sobre HIV/VIH/Aids/Sida: intensificando nossos esforços para eliminar o HIV/VIH/Aids/Sida. [internet]. Resolução adotada pela Assembleia Geral em 10 de junho de 2011. 2011 [acesso out. 2012]. Disponível: http://www.unaids.org.br/biblioteca/ Declara\%E7\%E3o\%20Pol\%EDtica\%20sobre\%20HIV\%20VIH\%20AIDS\%20SIDA\%202011\%20 PORTUGU\%CAS.pdf 
44. Greco DB, Simão M. Brazilian policy of universal acesso to Aids treatment: sustainability challenges and perspectives. Aids. 2007;21(supplement):S37-S45.

45. Figueiredo MAC. Dialogando com Freire e Boaventura sobre emancipação humana, multiculturalismo e educação popular. In: V Colóquio Internacional Paulo Freire; $19-22$ set. 2005; Recife, PE. [Internet]. [acesso out 2012]. Disponível: http://www.paulofreire.org.br/ pdf/comunicacoes_orais/DIALOGANDO\%20COM\%20FREIRE\%20E\%20BOAVENTURA\%20 SOBRE\%20EMANCIPA\%C3\%87\%C3\%830\%20HUMANA,\%20MULTICULTURALISMO\%20E\%20 EDUCA\%C3\%87\%C3\%830\%20POPULAR.pdf

46. Marx K. A questão judaica. $2^{\mathrm{a}}$ ed. São Paulo: Moraes; 1991

47. Freire P. Discussões em torno da pós-modernidade. In: Freire AMA, organizadora. Pedagogia dos sonhos possíveis. São Paulo: Editora Unesp; 2001.

48. United Nations. Adopting consensus text. General Assembly encourages member states to plan, pursue transition of national health care systems towards universal coverage. $67^{\text {a }}$ General Assembly; 12 dec. 2012. Disponível: http://www.un.org/News/Press/docs/2012/ga11326.doc. htm

49. Thucydites. The history of the Peloponesian wars. London: Penguin Classics; 1974.

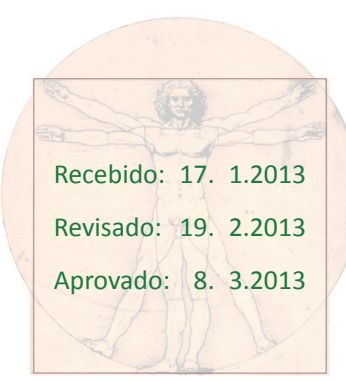

\section{Anexo}

Quadro 1. Circunstâncias selecionadas nas quais o ensaio clínico não deve ser realizado ${ }^{18}$

- Quando não existir capacidade local para conduzir uma avaliação científica e ética adequada e independente.

- Quando não puderem ser obtidos a participação realmente voluntária e o consentimento livre e esclarecido.

- Em locais onde as condições claramente puderem aumentar ou potencializ ar a vulnerabilidade ou facilitar a exploração.

- Quando não forem alcançados acordos entre os interessados na pesquisa quanto aos padrões de prevenção, questões de confidencialidade e acesso a cuidados e tratamentos médicos.

- Quando não forem alcançados acordos quanto a responsabilidades e planos para a disponibilização do produto da pesquisa (drogas, medidas preventivas ou procedimentos) que seja comprovadamente seguro, eficaz e acessível financeiramente às comunidades e países onde foi testado.

Fonte: WHO guidance on ethics of tuberculosis prevention, care and control. 